\title{
THE EFFECT OF WATER EXTRACTS FROM LEAVES OF Festuca rubra, F. ovina AND F. arundinacea ON THE INITIAL GROWTH AND DEVELOPMENT OF OTHER GRASS SPECIES
}

\author{
Halina Lipińska, Magdalena Sykut, Wanda Harkot \\ Department of Grassland and Landscape Forming, University of Life Sciences in Lublin \\ Akademicka 15, 20-950 Lublin, Poland \\ e-mail: hllpl@yahoo.com
}

Received: 12.10.2012

\begin{abstract}
The allelopathic effect of plants is one of the least known factors determining the stability of lawn swards. Leaves are a rich source of allelopathic substances. Washed out by rain or dew drops, or released during biomass decomposition, these substances can impact plants. In practice, cut sward is often left on the lawn surface and can have an allelopathic effect on regrowing plants. The effect of released allelochemicals depends on many factors, including their concentration. Hence, in order to maintain the high functional properties of the lawn, information is needed on the critical concentrations of allelochemicals inhibiting plant growth and development. Laboratory research was thus undertaken (on Petri dishes) to evaluate the effect of various water extracts of leaves of selected lawn grass cultivars. The following cultivars were the donors: 'Areta', 'Nimba', 'Olivia' (Festuca rubra); 'Espro', 'Pintor' ( $F$. ovina), and 'Asterix' ( $F$. arundinacea), while the acceptors were: 'Niwa' (Agrostis capillaris), 'Asterix' (Festuca arundinacea), 'Espro' (F. ovina), 'Areta' (F. rubra), 'Stadion' (Lolium perenne), and 'Bila' (Poa pratensis) - the species frequently sown in lawns. In the control treatments, distilled water was applied to the substrate.

The experiment revealed that the effect of water extracts of leaves varied depending on their concentration and donor variety as well as the sensitivity of the acceptor (the test plant). In comparison with the control treatments, the strongest negative impact was caused by the cultivars 'Olivia' ( $F$. rubra) and 'Pintor' ( $F$. ovina), followed by 'Asterix' $(F$. arundinacea). Among the acceptors, the greatest sensitivity to the presence of allelochemicals was shown by $A$. capillaris, and the smallest by F. arundinacea.
\end{abstract}

Key words: allelopathy, lawn grass cultivars, Festuca, initial growth and development

\section{INTRODUCTION}

The persistence and aesthetic value of a lawn depend on the floristic species composition of the sward that is influenced by the way the so-called bearing layer is prepared, the species and variety composition of the seed mixture used, the sensitivity of the components to habitat stress factors as well as a number of maintenance measures carried out each year, particularly cutting ( $\mathrm{Czarnecki}$ and $\mathrm{Harkot}$, 2002). In practice, after being cut (at varying height), the sward is often left on the lawn surface and can have an allelopathic effect on regrowing plants. It is one of the least researched factors determining the stability of lawn swards. Leaves are a rich source of allelopathic substances (Chung and Miller, 1995; Lipiń$\mathrm{sk} \mathrm{a}$ and $\mathrm{H}$ a r k o t, 2005). Washed out by rain or dew drops, or released during biomass decomposition, these substances can impact plants. The effect of released allelochemicals depends on many factors, including their concentration. At low concentrations, they show a stimulating effect, while at high concentrations their effect is inhibitory (Politycka and Lipińska, 2005). Hence, information on the critical concentrations of allelochemicals inhibiting plant growth and development can be important ( $\mathrm{H}$ a r k o t et al. 2000; Li pińska and Harkot, 2007).

In Poland's climate and soil conditions, the persistence and appearance of lawns is guaranteed by lawn cultivars of both Festuca rubra and Festuca ovina (H a r k o t et al. 2006). They are used in mixtures intended for establishing ornamental, park, recreational and sports ground lawns as well as for turfing of road 
embankments, roadsides, car parks, post-industrial and reclaimed areas (P a trz a ł e k, 2000; W o lski et al. 2006). More and more often, lawn cultivars of Festuca arundinace $a$ are also used to turf soils in difficult habitat conditions ( $\mathrm{Cz} \mathrm{y} \mathrm{ż} \mathrm{and} \mathrm{K} \mathrm{i} \mathrm{t} \mathrm{c} \mathrm{z} \mathrm{a} \mathrm{k,} \mathrm{2009).}$

The present study was undertaken to explain whether water extracts from the aboveground vegetative shoots of selected lawn cultivars of Festuca rubra, $F$. ovina and $F$. arundinacea have an impact on the initial growth and development of lawn cultivars of other grass species.

\section{MATERIALS AND METHODS}

The study was conducted at the Department of Grassland and Landscape Forming, University of Life Sciences in Lublin, in the years 2007-2010. A laboratory experiment was carried out to assess the impact of various concentrations of water extracts from aboveground vegetative shoots of donor grasses on the seed germination capacity as well as on root length and seedling height of acceptor grasses. The following cultivars were the donors: 'Areta', 'Nimba', and 'Olivia' (Festuca rubra); 'Espro' and 'Pintor' (F. ovina) as well as 'Asterix' ( $F$. arundinacea $)$, while the acceptors were: 'Niwa' (Agrostis capillaris), 'Asterix' (Festuca arundinacea), 'Espro' (F. ovina), 'Areta' (F. rubra), 'Stadion' (Lolium perenne), and 'Bila' (Poa pratensis) - species frequently sown in lawns.

Water extracts were made from aboveground vegetative shoots of the studied grass cultivars, collected at the propagation stage. The leaves were washed with running water to remove soil and other impurities, and then dried (with hot air) and cut into fine pieces (about $2 \mathrm{~mm}$ ). For biotests, three concentrations: 1.58, 2.35 , and $4.76 \%$, of water leachates from donor leaves were prepared by soaking, respectively, 15.8, 23.5 and $47.6 \mathrm{~g}$ of dry leaves, each portion in 1.01 water. After $24 \mathrm{~h}$, the solution was filtered through a filter. The leachates were stored at $5^{\circ} \mathrm{C}$.

Seeds of the acceptors (20 pieces of each) were placed on Petri dishes (in four replications) on three layers of chromatography paper (Whatman No. 3001917). $3 \mathrm{ml}$ of water extracts (donors) at appropriate concentrations were applied to the paper every day. In the control treatments, distilled water was applied to the paper. The germination capacity of A. capillaris seeds was assessed after 10 days, for $L$. perenne and $F$. arundinacea after 14 days, $F$. ovi$n$ a and $F$. rubra after 21 days, and $P$. pratensis after 28 days, according to the guidelines of $\mathrm{D}$ or $\mathrm{y} \mathrm{w}$ a $1 \mathrm{~s} \mathrm{k} \mathrm{i}$ et al. (1964). The results were expressed as the percentage of germinated seeds. On the same days when the seed germination capacity was determined, the length of the root system and the height of the seedlings were measured. These parameters were expressed in centimetres.

Biotests were conducted in the conditions of daily 12 -hour (7.00 am to $7.00 \mathrm{pm}$ ) artificial lighting with a SON-T Agro type high pressure output lamp (average light intensity of approx. 3000 lux at table level). Its special SGR 1400-type socket guaranteed appropriate and evenly distributed lighting of plants ( $\mathrm{U}$ of about $80 \%$ ). The air temperature in the room was optimal for the development of grass and ranged between 22 and $25{ }^{\circ} \mathrm{C}$.

The results were processed statistically using the SAS v.91 program, with the use of single-factor variance analysis and Tukey's multiple test. The means were divided into statistically uniform groups, and the significance level of $\leq 0.05$ was adopted. Mean values between which there are no statistically significant differences are marked with the same letter. The tables contain mean values from the years 2007-2010.

\section{RESULTS}

A. capillaris seeds subjected to the impact of water extracts from nearly all the donors under study showed poorer germination performance than in the control treatment. 'Espro' F. ovina extracts were an exception: they had a stimulating effect but it was insignificant in comparison with the control treatment (Table 1).

The greatest inhibitory effect, regardless of concentration, was shown by extracts from 'Olivia' F. rubra. The seed germination capacity significantly lower than in the control treatments was also observed in the case of extracts from 'Nimba' $F$. rubra, particularly those with the highest concentration $(4.76 \%)$. Water extracts from 'Areta' F. rubra and 'Pintor' F. ovina, but only with the highest concentration, also significantly inhibited the acceptor's seed germination. Water extracts from all donors had an adverse effect on root length and seedling height of A. capillaris (Table 1). Significantly shorter roots and smaller seedlings of the acceptor were observed after applying each extract; the greatest differences were caused by the highest concentrations $(4.76 \%)$. Regardless of their concentration, the greatest adverse effect was demonstrated by extracts from 'Asterix' $F$. arundinacea, followed by 'Areta' and 'Olivia' $F$. rubra, as they caused nearly $50 \%$ inhibition of root growth of A. capillaris.

Water extracts of all cultivars (except 'Espro') had an adverse impact on the seed germination capacity of $F$. arundinacea (Table 2). However, only extracts from 'Nimba' (already at a concentration of 2.38\%) as well as from 'Olivia' and 'Areta' F. rubra (at the highest concentration) had a significantly adverse 
impact in comparison with the control treatment where distilled water was applied to the paper. In the treatments where water extracts from 'Espro' and 'Pintor' $F$. ovina as well as from 'Asterix' $F$. arundinacea (at various concentrations) were applied, the seed germination capacity of $F$. arundinacea did not vary significantly. Extracts from 'Areta' F. rubra were an exception as their lowest concentration $(1.58 \%)$ significantly stimulated the seed germination of the acceptor. Regardless of the concentration, the roots of $F$. arundinacea were significantly shorter than in the control treatments only in the treatments with 'Olivia' $F$. rubra and 'Asterix' $F$. arundinacea (Table 2). The adverse effect of donors on the root length of seedlings of the acceptor was observed mainly after the application of extracts at the highest concentration. Extracts from 'Asterix' were an exception because, regardless of their concentration, they significantly inhibited root growth. $F$. arundinacea seedlings exhibited a greater sensitivity. Only extracts from itself ('Asterix' F. arundinacea) and extracts from 'Nimba' $F$. rubra slightly stimulated seedling height, while the other extracts had a significantly adverse effect on the process. Similarly to the roots, seedlings were significantly smaller than in the control treatments usually as a result of applying extracts at the highest concentration (4.76\%), and only in the case of extracts from 'Areta' F. rubra and 'Pintor' $F$. ovina at the concentration of $2.38 \%$.

Regardless of the concentration, the greatest adverse impact on seed germination of $F$. ovina was observed in the treatments where extracts from leaves of the cultivars 'Pintor' and 'Espro' and two F. rubra cultivars, 'Nimba' and 'Olivia', were applied (Table 3 ).

No significant differences were caused by extracts from 'Areta' F. rubra and 'Asterix' F. arundinacea, under whose influence the seed germination capacity of the acceptor was actually slightly higher than in the control treatment. As regards the impact of concentrations of water extracts from the particular donors, it was found that water extracts from 'Pintor' $F$. ovina, regardless of their concentration, caused significantly poorer seed germination, similarly to extracts from nearly all donors at the highest concentration $(4.76 \%)$. Extracts from 'Asterix' $F$. arundinacea were an exception because, regardless of their concentration, they only slightly inhibited the germination capacity of $F$. ovina. The root length of $F$. ovina also varied under the influence of extracts and distilled water (control treatment). In comparison with this treatment, all donors at each of the concentrations used (except the $1.58 \%$ concentration of extracts from 'Nimba') caused significant inhibition of the root growth of this acceptor (Table 3). However, the shortest roots were observed in seedlings where extracts from 'Olivia' $F$. rubra and 'Asterix' $F$. arundinacea were applied. The response of the seedlings, on the other hand, was quite different. Compared to the control treatment, none of the cultivars used, regardless of the concentration, caused significant differences in seedling height of the acceptor.

The germination of $F$. rubra seeds as influenced by all donors was poorer in comparison with the application of distilled water (Table 4). 'Asterix' $F$. arundinacea had the greatest inhibitory effect. Significant differences in seed germination were observed particularly in the treatments where extracts at the highest concentration (4.76\%) were used. Extracts from 'Nimba' $F$. rubra were an exception, because they did not cause the inhibition of seed germination of the acceptor, regardless of their concentration; the same effect occurred in the case of extracts from the other donors applied at the concentration of $1.58 \%$ and $2.38 \%$. Water extracts from the aboveground vegetative shoots of nearly all donors caused significant inhibition of the root growth of $F$. rubra seedlings (Table 4). Extracts from 'Areta' and 'Nimba F. rubra were an exception because the inhibition was only caused by their highest concentration, whereas at low concentrations these extracts caused the roots to be even longer than in the control treatment. Extracts from 'Asterix' $F$. arundinacea turned out to be the strongest inhibitor of root growth (at each of the concentrations used), as they caused the roots to be half as long as in the control treatment. In addition, the roots of this acceptor were adversely impacted by all concentrations of water extracts from 'Pintor' $F$. ovina and 'Olivia' $F$. rubra. On the other hand, the influence of donors on seedling height of $F$. rubra was insignificant. Regardless of the concentration, significant differences were found only in the case of extracts from 'Nimba' F. rubra, under whose influence seedlings were taller than in the control treatment (Table 4). Acceptor seedlings were found to be smaller only under the influence of the highest concentration $(4.76 \%)$ of extracts from 'Areta' F. rubra and 'Pintor' F. ovina.

In comparison with the control treatment, the germination capacity of $L$. perenne seeds was significantly lower under the influence of water extracts from 'Pintor' $F$. ovina and 'Asterix' $F$. arundinacea, regardless of their concentration, and extracts from the other donors (except 'Espro') at the highest concentration (4.76\%) (Table 5). Extracts from the other cultivars, applied at the lowest concentration, actually stimulated the germination of acceptor seeds, but only extracts from 'Espro' $F$. ovina had a significant impact. Water extracts from the aboveground vegetative shoots of all the grass cultivars resulted in 
significantly shorter roots of $L$. perenne in comparison with the control treatment. The strongest inhibitors of root growth were extracts from 'Olivia' F. rubra, 'Espro' $F$. ovina and 'Asterix' $F$. arundinacea (at each of the concentrations used). Similar patterns were also observed in the treatments where extracts from 'Areta' F. rubra and 'Pintor' F. ovina were applied. The seedling height of $L$. perenne, as influenced by the extracts, did not vary significantly from the control treatment. Regardless of the concentration, significantly smaller acceptor seedlings were only found in the treatments where extracts from 'Asterix' F. arundinacea and 'Olivia' F. rubra were applied at the highest concentration.

The germination capacity of $P$. pratensis seeds in the treatments subjected to water extracts from the aboveground vegetative shoots of all donor grasses was significantly lower than in the control treatment (Table 6).

Under the influence of each extract concentration (regardless of the changing donor), $P$. pratensis seeds showed significantly poorer germination than under distilled water conditions. However, such differences were not observed between the concentrations of $1.58 \%, 2.38 \%$ and $4.76 \%$, with the exception of extracts from 'Nimba' $F$. rubra which, at the concentration of $4.76 \%$, inhibited the process to the greatest extent. In comparison with the control treatment, the water extracts from all donors significantly inhibited the growth of roots in $P$. pratensis seedlings. Regardless of the variety, each of the concentrations used caused significant inhibition: the strongest inhibition occurred at the concentration of $4.76 \%$. The seedling height of $P$. pratensis was significantly inhibited by all extracts from 'Pintor' $F$. ovina and 'Asterix' $F$. arundinacea as well as extracts from most donors at the concentration of $4.76 \%$. Only the highest concentrations of extracts from 'Nimba' $F$. rubra did not cause significant differences. No significant influence on acceptor seedling height was observed in the case of extracts from 'Areta', 'Nimba' and 'Olivia' F. rubra applied at the concentration of $1.58 \%$ and $2.38 \%$.

Table 1

Seed germination capacity, root length and seedling height of Agrostis capillaris subjected to various concentrations of water extracts from aboveground vegetative shoots of lawn grass cultivars - donors

\begin{tabular}{|c|c|c|c|c|c|c|c|}
\hline \multirow{3}{*}{ 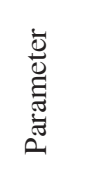 } & \multirow{3}{*}{$\begin{array}{c}\text { Extract } \\
\text { concentrations }\end{array}$} & \multicolumn{6}{|c|}{ Donors } \\
\hline & & \multicolumn{3}{|c|}{ F. rubra } & \multicolumn{2}{|c|}{ F. ovina } & \multirow{2}{*}{$\begin{array}{c}\text { F. arundinacea } \\
\text {,Asterix' }\end{array}$} \\
\hline & & ,Areta' & ,Nimba' & ,Olivia' & „Espro’ & ,Pintor' & \\
\hline \multirow{5}{*}{ 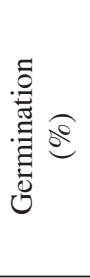 } & 0 & $64.6 \mathrm{a}$ & $64.6 \mathrm{a}$ & $64.6 \mathrm{a}$ & $64.6 a b$ & $64.6 \mathrm{a}$ & $64.6 \mathrm{ab}$ \\
\hline & $1.58 \%$ & $73.9 \mathrm{a}$ & $66.7 \mathrm{a}$ & $46.9 b$ & $79.2 \mathrm{a}$ & $72.9 a$ & $71.8 \mathrm{a}$ \\
\hline & $2.38 \%$ & $61.4 \mathrm{ab}$ & $51 \mathrm{ab}$ & $37.5 b c$ & $70.8 \mathrm{ab}$ & $64.6 \mathrm{a}$ & $63.5 \mathrm{ab}$ \\
\hline & $4.76 \%$ & $45.8 b$ & $42.7 b$ & $30.2 \mathrm{c}$ & $60.4 b$ & $43.7 b$ & $45.8 b$ \\
\hline & Mean for donor & $61.4 \mathrm{ab}$ & $56.2 \mathrm{~b}$ & $44.8 \mathrm{c}$ & $68.7 \mathrm{a}$ & $61.4 \mathrm{ab}$ & $61.4 \mathrm{ab}$ \\
\hline \multirow{5}{*}{ 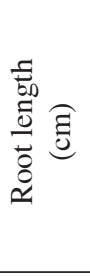 } & 0 & $0.9 \mathrm{a}$ & $0.9 \mathrm{a}$ & $0.9 \mathrm{a}$ & $0.9 \mathrm{a}$ & $0.9 \mathrm{a}$ & $0.9 \mathrm{a}$ \\
\hline & $1.58 \%$ & $0.3 b$ & $0.6 \mathrm{~b}$ & $0.5 \mathrm{~b}$ & $0.5 \mathrm{~b}$ & $0.6 \mathrm{~b}$ & $0.4 \mathrm{~b}$ \\
\hline & $2.38 \%$ & $0.3 b$ & $0.3 \mathrm{c}$ & $0.3 \mathrm{c}$ & $0.4 \mathrm{c}$ & $0.4 \mathrm{c}$ & $0.3 \mathrm{c}$ \\
\hline & $4.76 \%$ & $0.3 b$ & $0.3 \mathrm{c}$ & $0.1 \mathrm{~d}$ & $0.3 \mathrm{c}$ & $0.3 \mathrm{c}$ & $0.2 \mathrm{c}$ \\
\hline & Mean for donor & $0.4 \mathrm{c}$ & $0.5 b$ & $0.4 \mathrm{c}$ & $0.5 b$ & $0.5 b$ & $0.4 \mathrm{c}$ \\
\hline \multirow{5}{*}{ 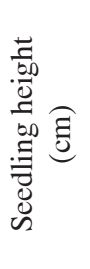 } & 0 & $2.1 \mathrm{a}$ & $2.1 \mathrm{a}$ & $2.1 \mathrm{a}$ & $2.1 \mathrm{a}$ & $2.1 \mathrm{a}$ & $2.1 \mathrm{a}$ \\
\hline & $1.58 \%$ & $1.7 \mathrm{~b}$ & $1.9 \mathrm{~b}$ & $1.7 \mathrm{~b}$ & $1.9 \mathrm{a}$ & $1.7 \mathrm{~b}$ & $1.7 \mathrm{~b}$ \\
\hline & $2.38 \%$ & $1.5 \mathrm{c}$ & $1.7 \mathrm{~b}$ & $1.6 \mathrm{~b}$ & $1.7 \mathrm{~b}$ & $1.9 \mathrm{a}$ & $1.3 \mathrm{c}$ \\
\hline & $4.76 \%$ & $1.0 \mathrm{~d}$ & $0.8 \mathrm{c}$ & $1.1 \mathrm{c}$ & $0.9 \mathrm{c}$ & $1.0 \mathrm{c}$ & $1.1 \mathrm{~d}$ \\
\hline & Mean for donor & $1.6 \mathrm{c}$ & $1.6 b c$ & $1.6 b c$ & $1.6 b c$ & $1.7 \mathrm{~b}$ & $1.5 \mathrm{c}$ \\
\hline
\end{tabular}

Explanation: Means followed by the same letter in column do not differ significantly. 
Table 2

Seed germination capacity, root length and seedling height of Festuca arundinacea subjected to various concentrations of water extracts from aboveground vegetative shoots of lawn grass cultivars - donors

\begin{tabular}{|c|c|c|c|c|c|c|c|}
\hline \multirow{3}{*}{ 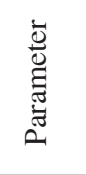 } & \multirow{3}{*}{$\begin{array}{c}\text { Extract } \\
\text { concentrations }\end{array}$} & \multicolumn{6}{|c|}{ Donors } \\
\hline & & \multicolumn{3}{|c|}{ F.rubra } & \multicolumn{2}{|c|}{ F.ovina } & \multirow{2}{*}{$\frac{\text { F.arundinacea }}{\text {,Asterix' }}$} \\
\hline & & ,Areta' & ,Nimba' & ,Olivia’ & „Espro’ & ,Pintor' & \\
\hline \multirow{5}{*}{ 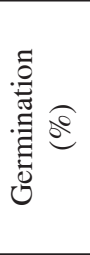 } & 0 & $70.8 b$ & $70.8 \mathrm{a}$ & $70.8 \mathrm{a}$ & $70.8 \mathrm{a}$ & $70.8 \mathrm{ab}$ & $70.8 \mathrm{a}$ \\
\hline & $1.58 \%$ & $85.4 \mathrm{a}$ & $58.3 \mathrm{ab}$ & $63.5 \mathrm{a}$ & $79.2 \mathrm{a}$ & $72.9 \mathrm{a}$ & $69.8 \mathrm{a}$ \\
\hline & $2.38 \%$ & $62.5 b c$ & $55.2 \mathrm{~b}$ & $58.3 \mathrm{a}$ & $78.1 \mathrm{a}$ & $64.6 \mathrm{ab}$ & $63.5 \mathrm{a}$ \\
\hline & $4.76 \%$ & $55.2 \mathrm{c}$ & $44.8 \mathrm{~b}$ & $41.7 \mathrm{~b}$ & $66.7 \mathrm{a}$ & $59.4 \mathrm{~b}$ & $60.4 \mathrm{a}$ \\
\hline & Mean for donor & $68.5 \mathrm{a}$ & $57.3 \mathrm{c}$ & $58.6 \mathrm{bc}$ & $73.7 \mathrm{a}$ & $66.9 \mathrm{ab}$ & $66.1 \mathrm{abc}$ \\
\hline \multirow{5}{*}{ 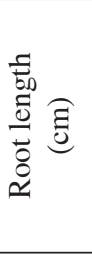 } & 0 & $2.4 \mathrm{bc}$ & $2.4 \mathrm{a}$ & $2.4 \mathrm{a}$ & $2.4 \mathrm{ab}$ & $2.4 \mathrm{a}$ & $2.4 \mathrm{a}$ \\
\hline & $1.58 \%$ & $3.2 \mathrm{a}$ & $2.9 \mathrm{a}$ & $2.3 \mathrm{a}$ & $2.7 \mathrm{a}$ & $2.7 \mathrm{a}$ & $1.6 b$ \\
\hline & $2.38 \%$ & $2.8 \mathrm{ab}$ & $2.4 \mathrm{a}$ & $2.3 \mathrm{a}$ & $2.1 \mathrm{bc}$ & $2.3 \mathrm{a}$ & $1.4 \mathrm{~b}$ \\
\hline & $4.76 \%$ & $1.9 \mathrm{c}$ & $1.4 \mathrm{~b}$ & $1.5 \mathrm{~b}$ & $1.7 \mathrm{c}$ & $1.4 \mathrm{~b}$ & $1.5 b$ \\
\hline & Mean for donor & $2.6 \mathrm{a}$ & $2.3 \mathrm{~b}$ & $2.1 \mathrm{~b}$ & $2.2 \mathrm{~b}$ & $2.2 \mathrm{~b}$ & $1.7 \mathrm{c}$ \\
\hline \multirow{5}{*}{ 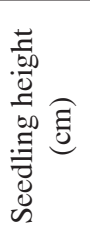 } & 0 & $6.1 \mathrm{a}$ & $6.1 \mathrm{ab}$ & $6.1 \mathrm{a}$ & $6.1 \mathrm{a}$ & $6.1 \mathrm{a}$ & $6.1 \mathrm{~b}$ \\
\hline & $1.58 \%$ & $5.9 \mathrm{ab}$ & $6.8 \mathrm{a}$ & $5.6 \mathrm{a}$ & $5.6 \mathrm{ab}$ & $5.4 \mathrm{ab}$ & $6.9 \mathrm{a}$ \\
\hline & $2.38 \%$ & $4.9 \mathrm{c}$ & $6.1 \mathrm{ab}$ & $5.3 \mathrm{a}$ & $6.0 \mathrm{a}$ & $5.1 \mathrm{~b}$ & $6.5 \mathrm{ab}$ \\
\hline & $4.76 \%$ & $5.0 \mathrm{bc}$ & $5.2 b$ & $5.2 \mathrm{a}$ & $5.0 \mathrm{~b}$ & $3.1 \mathrm{c}$ & $5.5 c$ \\
\hline & Mean for donor & $5.5 \mathrm{c}$ & $6.0 \mathrm{a}$ & $5.5 \mathrm{c}$ & $5.7 \mathrm{bc}$ & $4.9 \mathrm{~d}$ & $6.2 \mathrm{a}$ \\
\hline
\end{tabular}

Explanation: Means followed by the same letter in column do not differ significantly.

Table 3

Seed germination capacity, root length and seedling height of Festuca ovina subjected to various concentrations of water extracts from aboveground vegetative shoots of lawn grass cultivars - donors

\begin{tabular}{|c|c|c|c|c|c|c|c|}
\hline \multirow{3}{*}{ 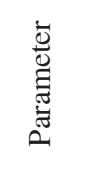 } & \multirow{3}{*}{$\begin{array}{c}\text { Extract } \\
\text { concentrations }\end{array}$} & \multicolumn{6}{|c|}{ Donors } \\
\hline & & \multicolumn{3}{|c|}{ F.rubra } & \multicolumn{2}{|c|}{ F.ovina } & \multirow{2}{*}{$\frac{\text { F.arundinacea }}{\text {,Asterix' }}$} \\
\hline & & ,Areta' & ,Nimba' & ,Olivia' & „Espro’ & ,Pintor' & \\
\hline \multirow{5}{*}{ 总 } & 0 & $87.5 \mathrm{a}$ & $87.5 \mathrm{a}$ & $87.5 \mathrm{a}$ & $87.5 \mathrm{a}$ & $87.5 \mathrm{a}$ & $87.5 \mathrm{a}$ \\
\hline & $1.58 \%$ & $86.3 \mathrm{a}$ & $91.7 \mathrm{a}$ & $87.7 \mathrm{a}$ & $80.2 \mathrm{ab}$ & $48.9 b$ & $72.9 \mathrm{a}$ \\
\hline & $2.38 \%$ & $72.9 \mathrm{~b}$ & $77 b$ & $76 a$ & $65.6 \mathrm{~b}$ & $61.4 \mathrm{~b}$ & $86.4 \mathrm{a}$ \\
\hline & $4.76 \%$ & $66.7 b$ & $54.2 \mathrm{~b}$ & $34.4 \mathrm{~b}$ & $23.9 \mathrm{c}$ & $47.9 b$ & $81.2 \mathrm{a}$ \\
\hline & Mean for donor & $78.3 \mathrm{ab}$ & $77.6 \mathrm{~b}$ & $71.4 \mathrm{bc}$ & $64.3 \mathrm{~cd}$ & $61.4 d$ & 82.0 \\
\hline \multirow{5}{*}{ 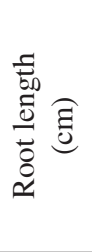 } & 0 & $5.3 \mathrm{a}$ & $5.3 \mathrm{a}$ & $5.3 \mathrm{a}$ & $5.3 \mathrm{a}$ & $5.3 \mathrm{a}$ & $5.3 b$ \\
\hline & $1.58 \%$ & $2.9 \mathrm{~b}$ & $4.8 \mathrm{a}$ & $3.0 \mathrm{~b}$ & $3.7 b$ & $3.5 b$ & $3.6 \mathrm{c}$ \\
\hline & $2.38 \%$ & $3.2 b$ & $3.7 \mathrm{~b}$ & $2.2 \mathrm{c}$ & $2.3 \mathrm{c}$ & $3.6 \mathrm{~b}$ & $2.1 \mathrm{c}$ \\
\hline & $4.76 \%$ & $2.8 \mathrm{~b}$ & $3.8 \mathrm{~b}$ & $1.3 \mathrm{~d}$ & $2.1 \mathrm{c}$ & $3.1 \mathrm{~b}$ & $2.2 \mathrm{c}$ \\
\hline & Mean for donor & $3.5 \mathrm{~cd}$ & $4.4 \mathrm{~b}$ & $2.9 \mathrm{~d}$ & $3.3 \mathrm{~cd}$ & $3.9 \mathrm{c}$ & $3.3 \mathrm{~cd}$ \\
\hline \multirow{5}{*}{ 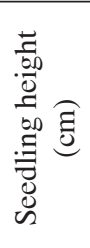 } & 0 & $6.9 \mathrm{a}$ & $6.9 \mathrm{a}$ & $6.9 \mathrm{~b}$ & $6.9 \mathrm{a}$ & $6.9 \mathrm{a}$ & $6.9 \mathrm{ab}$ \\
\hline & $1.58 \%$ & $6.5 \mathrm{a}$ & $6.7 \mathrm{a}$ & $8.7 \mathrm{a}$ & $6.9 \mathrm{a}$ & $7.0 \mathrm{a}$ & $7.1 \mathrm{a}$ \\
\hline & $2.38 \%$ & $7.6 \mathrm{a}$ & $6.4 \mathrm{a}$ & $6.7 b$ & $7.2 \mathrm{a}$ & $7.4 \mathrm{a}$ & $6.6 \mathrm{ab}$ \\
\hline & $4.76 \%$ & $6.7 \mathrm{a}$ & $6.3 \mathrm{a}$ & $6.7 b$ & $6.8 \mathrm{a}$ & $6.8 \mathrm{a}$ & $6.2 \mathrm{~b}$ \\
\hline & Mean for donor & $6.9 \mathrm{ab}$ & $6.6 \mathrm{~b}$ & $7.2 \mathrm{a}$ & $6.9 \mathrm{ab}$ & 7.0ab & $6.7 b$ \\
\hline
\end{tabular}

Explanation: Means followed by the same letter in column do not differ significantly. 
Table 4

Seed germination capacity, root length and seedling height of Festuca rubra subjected to various concentrations of water extracts from aboveground vegetative shoots of lawn grass cultivars - donors

\begin{tabular}{|c|c|c|c|c|c|c|c|}
\hline \multirow{3}{*}{ 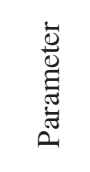 } & \multirow{3}{*}{$\begin{array}{c}\text { Extract } \\
\text { concentrations }\end{array}$} & \multicolumn{6}{|c|}{ Donors } \\
\hline & & \multicolumn{3}{|c|}{ F.rubra } & \multicolumn{2}{|c|}{ F.ovina } & \multirow{2}{*}{$\begin{array}{c}\text { F.arundinacea } \\
\text {,Asterix' }\end{array}$} \\
\hline & & ,Areta' & ,Nimba' & ,Olivia’ & ,Espro' & ,Pintor' & \\
\hline \multirow{5}{*}{ 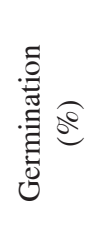 } & 0 & $94.8 \mathrm{a}$ & $94.8 \mathrm{a}$ & $94.8 \mathrm{a}$ & $94.8 \mathrm{a}$ & $94.8 \mathrm{a}$ & $94.8 \mathrm{a}$ \\
\hline & $1.58 \%$ & $96.9 \mathrm{a}$ & $97.9 \mathrm{a}$ & $85.4 \mathrm{a}$ & $96.9 \mathrm{a}$ & $86.4 \mathrm{a}$ & $69.8 \mathrm{ab}$ \\
\hline & $2.38 \%$ & $81.2 \mathrm{ab}$ & $95.8 \mathrm{a}$ & $83.3 \mathrm{ab}$ & $92.7 \mathrm{a}$ & $86.4 \mathrm{a}$ & 73.9ab \\
\hline & $4.76 \%$ & $65.6 b$ & $85.4 \mathrm{a}$ & $65.6 \mathrm{~b}$ & $71.9 \mathrm{~b}$ & $63.5 b$ & $64.6 b$ \\
\hline & Mean for donor & $84.6 \mathrm{~b}$ & $93.5 \mathrm{a}$ & $82.3 b c$ & $89.1 \mathrm{ab}$ & $83.6 \mathrm{~b}$ & $75.8 \mathrm{c}$ \\
\hline \multirow{5}{*}{ 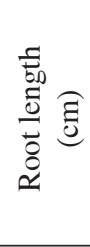 } & 0 & $8.0 \mathrm{~b}$ & $8.0 \mathrm{~b}$ & $8.0 \mathrm{~b}$ & $8.0 \mathrm{~b}$ & $8.0 \mathrm{~b}$ & $8.0 \mathrm{~b}$ \\
\hline & $1.58 \%$ & $7.3 \mathrm{~b}$ & $8.3 \mathrm{a}$ & $5.1 \mathrm{~b}$ & $6.0 \mathrm{ab}$ & $6.6 \mathrm{~b}$ & $3.5 b$ \\
\hline & $2.38 \%$ & $9.4 \mathrm{a}$ & $6.6 b$ & $4.2 \mathrm{bc}$ & $3.8 \mathrm{~b}$ & $5.6 \mathrm{~b}$ & $2.8 \mathrm{c}$ \\
\hline & $4.76 \%$ & $3.2 \mathrm{c}$ & $4.4 \mathrm{c}$ & $3.2 \mathrm{c}$ & $3.8 \mathrm{~b}$ & $2.5 \mathrm{c}$ & $2.4 \mathrm{c}$ \\
\hline & Mean for donor & $7.0 \mathrm{a}$ & $6.8 \mathrm{a}$ & $5.1 \mathrm{~b}$ & $5.4 \mathrm{~b}$ & $5.7 \mathrm{~b}$ & $4.2 \mathrm{c}$ \\
\hline \multirow{5}{*}{ 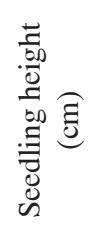 } & 0 & $7.2 \mathrm{a}$ & $7.2 \mathrm{a}$ & $7.2 \mathrm{a}$ & $7.2 \mathrm{a}$ & $7.2 \mathrm{a}$ & $7.2 \mathrm{a}$ \\
\hline & $1.58 \%$ & $7.0 \mathrm{~b}$ & $7.6 a$ & $7.8 \mathrm{a}$ & $7.1 \mathrm{a}$ & $8.0 \mathrm{a}$ & $7.5 \mathrm{a}$ \\
\hline & $2.38 \%$ & $7.8 \mathrm{a}$ & $7.7 \mathrm{a}$ & $7.5 \mathrm{ab}$ & $7.4 \mathrm{a}$ & $7.8 \mathrm{a}$ & $6.9 \mathrm{a}$ \\
\hline & $4.76 \%$ & $6.0 \mathrm{c}$ & $7.5 \mathrm{a}$ & $6.8 \mathrm{~b}$ & $7.2 \mathrm{a}$ & $5.2 b$ & $6.8 \mathrm{a}$ \\
\hline & Mean for donor & $7.0 \mathrm{~b}$ & $7.5 \mathrm{a}$ & $7.3 \mathrm{ab}$ & $7.2 \mathrm{ab}$ & $7.0 \mathrm{~b}$ & 7.1ab \\
\hline
\end{tabular}

Explanation: Means followed by the same letter in column do not differ significantly.

Table 5

Seed germination capacity, root length and seedling height of Lolium perenne subjected to various concentrations of water extracts from aboveground vegetative shoots of lawn grass cultivars - donors

\begin{tabular}{|c|c|c|c|c|c|c|c|}
\hline \multirow{3}{*}{ 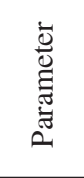 } & \multirow{3}{*}{$\begin{array}{c}\text { Extract } \\
\text { concentrations }\end{array}$} & \multicolumn{6}{|c|}{ Donors } \\
\hline & & \multicolumn{3}{|c|}{ F.rubra } & \multicolumn{2}{|c|}{ F.ovina } & \multirow{2}{*}{$\begin{array}{c}\text { F.arundinacea } \\
\text {,Asterix' }\end{array}$} \\
\hline & & ,Areta' & ,Nimba' & ,Olivia' & ,Espro' & ,Pintor' & \\
\hline \multirow{5}{*}{ 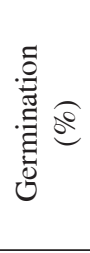 } & 0 & $81.2 \mathrm{ab}$ & $81.2 \mathrm{a}$ & $81.2 \mathrm{a}$ & $81.2 \mathrm{~b}$ & $81.2 \mathrm{a}$ & $81.2 \mathrm{a}$ \\
\hline & $1.58 \%$ & $90.6 \mathrm{a}$ & $88.5 \mathrm{a}$ & $93.7 \mathrm{a}$ & $94.8 \mathrm{a}$ & $83.3 \mathrm{a}$ & $79.2 \mathrm{ab}$ \\
\hline & $2.38 \%$ & $89.6 \mathrm{a}$ & $85.4 \mathrm{a}$ & $90.6 \mathrm{a}$ & $87.5 \mathrm{ab}$ & $64.6 b$ & $61.4 b c$ \\
\hline & $4.76 \%$ & $71.9 \mathrm{~b}$ & $59.4 b$ & $64.6 \mathrm{~b}$ & $79.2 \mathrm{~b}$ & $55.2 b$ & $47.9 \mathrm{c}$ \\
\hline & Mean for donor & $83.3 \mathrm{a}$ & $78.6 \mathrm{a}$ & $82.5 \mathrm{a}$ & $85.7 \mathrm{a}$ & $71.1 \mathrm{~b}$ & $67.4 \mathrm{c}$ \\
\hline \multirow{5}{*}{ 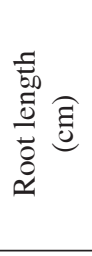 } & 0 & $5.3 \mathrm{a}$ & $5.3 \mathrm{a}$ & $5.3 \mathrm{a}$ & $5.3 \mathrm{a}$ & $5.3 \mathrm{a}$ & $5.3 \mathrm{a}$ \\
\hline & $1.58 \%$ & $2.9 \mathrm{~b}$ & $4.8 \mathrm{a}$ & $3.0 \mathrm{~b}$ & $3.7 \mathrm{~b}$ & $3.6 \mathrm{~b}$ & $3.6 \mathrm{~b}$ \\
\hline & $2.38 \%$ & $3.2 \mathrm{~b}$ & $3.7 \mathrm{~b}$ & $2.2 \mathrm{c}$ & $2.3 \mathrm{c}$ & $3.5 \mathrm{~b}$ & $2.1 \mathrm{c}$ \\
\hline & $4.76 \%$ & $2.8 b$ & $3.8 \mathrm{~b}$ & $1.3 \mathrm{~d}$ & $2.1 \mathrm{c}$ & $3.1 \mathrm{~b}$ & $2.2 \mathrm{c}$ \\
\hline & Mean for donor & $3.5 \mathrm{~cd}$ & $4.4 \mathrm{~b}$ & $2.9 \mathrm{e}$ & $3.3 \mathrm{de}$ & $3.9 \mathrm{c}$ & $3.3 \mathrm{de}$ \\
\hline \multirow{5}{*}{ 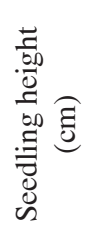 } & 0 & $6.9 \mathrm{ab}$ & $6.9 \mathrm{ab}$ & $6.9 \mathrm{ab}$ & $6.9 \mathrm{ab}$ & $6.9 \mathrm{ab}$ & $6.9 \mathrm{ab}$ \\
\hline & $1.58 \%$ & $6.7 \mathrm{a}$ & $6.7 \mathrm{a}$ & $8.7 \mathrm{a}$ & $6.9 \mathrm{a}$ & $7.4 \mathrm{a}$ & $7.1 \mathrm{a}$ \\
\hline & $2.38 \%$ & $7.6 \mathrm{a}$ & $6.4 \mathrm{a}$ & $6.8 b$ & $7.2 \mathrm{a}$ & $7.0 \mathrm{a}$ & $6.6 \mathrm{ab}$ \\
\hline & $4.76 \%$ & $6.5 \mathrm{a}$ & $6.3 \mathrm{a}$ & $6.0 \mathrm{c}$ & $6.8 \mathrm{a}$ & $6.8 \mathrm{a}$ & $6.2 b$ \\
\hline & Mean for donor & $6.9 \mathrm{ab}$ & $6.6 \mathrm{~b}$ & $7.1 \mathrm{a}$ & $6.9 \mathrm{ab}$ & 7.0ab & $6.7 \mathrm{c}$ \\
\hline
\end{tabular}

Explanation: Means followed by the same letter in column do not differ significantly. 
Table 6

Seed germination capacity, root length and seedling height of Poa pratensis subjected to various concentrations of water extracts from aboveground vegetative shoots of lawn grass cultivars - donors

\begin{tabular}{|c|c|c|c|c|c|c|c|}
\hline \multirow{3}{*}{$\begin{array}{l}\dot{\bar{d}} \\
\bar{\Xi} \\
\overline{\tilde{J}} \\
a\end{array}$} & \multirow{3}{*}{$\begin{array}{c}\text { Extract } \\
\text { concentrations }\end{array}$} & \multicolumn{6}{|c|}{ Donors } \\
\hline & & \multicolumn{3}{|c|}{ F.rubra } & \multicolumn{2}{|c|}{ F.ovina } & \multirow{2}{*}{$\begin{array}{c}\text { F.arundinacea } \\
\text {,Asterix' }\end{array}$} \\
\hline & & ,Areta' & ,Nimba' & ,Olivia' & „Espro’ & ,Pintor' & \\
\hline \multirow{5}{*}{ 离 } & 0 & $77.1 \mathrm{a}$ & $77.1 \mathrm{a}$ & $77.1 \mathrm{a}$ & $77.1 \mathrm{a}$ & $77.1 \mathrm{a}$ & $77.1 \mathrm{a}$ \\
\hline & $1.58 \%$ & $55.2 \mathrm{~b}$ & $62.5 b$ & $52.1 \mathrm{~b}$ & $48.9 \mathrm{~b}$ & $53.1 \mathrm{~b}$ & $47.9 \mathrm{~b}$ \\
\hline & $2.38 \%$ & $55.2 b$ & $56.2 b$ & $49.1 b$ & $57.3 b$ & $46.9 \mathrm{~b}$ & $47.9 \mathrm{~b}$ \\
\hline & $4.76 \%$ & $53.1 \mathrm{~b}$ & $41.6 \mathrm{c}$ & $35.4 \mathrm{~b}$ & $46.9 b$ & $41.7 \mathrm{~b}$ & $38.5 b$ \\
\hline & Mean for donor & $60.1 \mathrm{ab}$ & $59.3 \mathrm{~b}$ & $53.4 \mathrm{c}$ & $57.5 \mathrm{~b}$ & $54.7 \mathrm{c}$ & $52.9 \mathrm{c}$ \\
\hline \multirow{5}{*}{$\underset{\substack{0 \\
\stackrel{0}{0}}}{\stackrel{0}{0}}$} & 0 & $1.5 \mathrm{a}$ & $1.5 \mathrm{a}$ & $1.5 \mathrm{a}$ & $1.5 \mathrm{a}$ & $1.5 \mathrm{a}$ & $1.5 \mathrm{a}$ \\
\hline & $1.58 \%$ & $1.1 \mathrm{~b}$ & $1.1 \mathrm{~b}$ & $1.0 \mathrm{~b}$ & $1.1 \mathrm{~b}$ & $1.3 \mathrm{a}$ & $1.0 \mathrm{~b}$ \\
\hline & $2.38 \%$ & $1.0 \mathrm{c}$ & $1.1 \mathrm{~b}$ & $1.7 \mathrm{a}$ & $0.7 \mathrm{c}$ & $0.5 b$ & $0.6 \mathrm{c}$ \\
\hline & $4.76 \%$ & $0.9 \mathrm{~d}$ & $1.0 \mathrm{~b}$ & $0.8 \mathrm{c}$ & $0.6 \mathrm{c}$ & $0.6 \mathrm{~b}$ & $0.5 \mathrm{c}$ \\
\hline & Mean for donor & $1.1 \mathrm{c}$ & $1.2 \mathrm{bc}$ & $1.2 \mathrm{~b}$ & $1.0 \mathrm{~d}$ & $1.0 \mathrm{~d}$ & $0.9 \mathrm{~d}$ \\
\hline \multirow{5}{*}{ 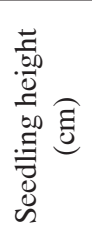 } & 0 & $3.6 \mathrm{a}$ & $3.6 \mathrm{a}$ & $3.6 \mathrm{a}$ & $3.6 \mathrm{~b}$ & $3.6 \mathrm{a}$ & $3.6 \mathrm{a}$ \\
\hline & $1.58 \%$ & $3.5 \mathrm{a}$ & $3.5 \mathrm{ab}$ & $3.9 \mathrm{a}$ & $3.9 \mathrm{a}$ & $3.2 \mathrm{~b}$ & $3.0 \mathrm{~b}$ \\
\hline & $2.38 \%$ & $3.4 \mathrm{a}$ & $3.6 \mathrm{ab}$ & $2.8 \mathrm{~b}$ & $3.3 \mathrm{c}$ & $3.0 \mathrm{c}$ & $3.0 \mathrm{~b}$ \\
\hline & $4.76 \%$ & $1.0 \mathrm{~b}$ & $3.3 b$ & 1.9 & $2.0 \mathrm{~d}$ & $2.7 \mathrm{~d}$ & $2.7 \mathrm{c}$ \\
\hline & Mean for donor & $2.9 \mathrm{~d}$ & $3.5 \mathrm{a}$ & $3.1 \mathrm{~cd}$ & $3.2 \mathrm{~b}$ & $3.1 \mathrm{bc}$ & $3.1 \mathrm{bc}$ \\
\hline
\end{tabular}

Explanation: Means followed by the same letter in column do not differ significantly.

\section{DISCUSSION}

One of the key stages of studying the allelopathic potential of plants is bioanalysis which typically assesses the degree of inhibition (more rarely the degree of stimulation) of growth and development processes of the test plants, including the seed germination capacity, root length and seedling height of the test species (B $1 \mathrm{um}, 1999)$. These parameters were also assessed in the present biotests, because the mutual interaction of plants begins with the germination of grass seeds and continues throughout their growth and development in a given community ( $\mathrm{L}$ i p i ń s k a and $\mathrm{H}$ a r k o $\mathrm{t}$, 2007). The assessment of the impact of water extracts from the aboveground vegetative shoots of lawn grass cultivars (donors) on the initial growth and development of lawn grass cultivars of other species (acceptors) revealed significant differences both in the strength of donor impact and in the response of the acceptors.

It was found that the growth of the test species was inhibited to a varying degree depending on the donor variety and extract concentration. The smallest degree of inhibition was observed in the case of seed germination, while the greatest in the case of root length. The growth of the acceptor's aboveground shoots was inhibited by water extracts to a lesser extent. It is worth remembering that seedling roots usually show a greater sensitivity to the presence of allelochemicals than the aboveground shoots, and within a specific bracket their growth is proportional to the concentration of substances ( $\mathrm{Lickfeld} \mathrm{t} \mathrm{et} \mathrm{al.} \mathrm{2001).} \mathrm{The} \mathrm{greater}$ sensitivity of the roots (than the aboveground shoots) to the impact of allelopathic substances was also confirmed in the studies by Il a m and B e g u m (2011).

Specific species and cultivars differ with regard to their capacity to synthesize allelochemicals and, by the same token, they have a different strength of impact (B a g he st an i et al. 1999). This was also confirmed by the present study where, regardless of the extract concentration, the smallest adverse impact on seed germination, root length and seedling height of the acceptor was demonstrated by 'Nimba' $F$. rubra. The strongest adverse impact was shown by 'Olivia' F. rubra and 'Pintor' F. ovina. The significant allelopathic potential of these species was also demonstrated in the study by B e rtin a et al. (2005). F. ovina and $F$. rubra - turf grasses commonly used in the USA and Europe - caused a significant inhibition of, among others, D. sanguinalis, T. repens and $T$. officinale. The allelopathic activity of $F$. rubra was also indicated in earlier studies by F a l k o w s k i (1958) who found significantly poorer germination of $P$. pratensis and D. glomerata seeds on water extracts from the soil 
where $F$. rubra grew in comparison with extracts from soil not covered with grass vegetation. The study of Majchrzak (2007) also indicated an adverse impact of the proximity of $F$. rubra on other plant species. A considerable allelopathic potential towards selected acceptors has also been demonstrated by 'Asterix' $F$. arundinacea. The literature mentions cases which confirm the allelopathic properties of $F$. arundinacea (Smith and Martin, 1994; Chung and Mille r, 1995). This donor's allelopathic activity towards other grass species was also indicated by $\mathrm{B} \mathrm{uta}$ and S p a u ld ing (1987) and Li p i ń s k a (2005). In these studies, water extracts from $F$. arundinacea leaves inhibited the initial growth of grasses and legumes.

The allelopathic potential of a given donor is determined by the concentration of biologically active compounds. As a rule, these compounds have a stimulating effect at low concentrations, and an inhibiting effect at high concentrations (O l e s z e k, 1992). Similar patterns were also found during the present biotests. Water extracts from aboveground vegetative shoots applied at the lowest concentration $(1.58 \%)$ had a positive impact on seed germination and initial growth of the test species-acceptors. Their significantly adverse impact was only manifested in the slower growth of the acceptor's roots, and this was only under the influence of 'Asterix' $F$. arundinacea, followed by Olivia F. rubra, 'Espro' and 'Pintor' F. ovina. All the parameters assessed under the influence of the highest concentration $(4.76 \%)$ of water extracts from nearly all donors were lower than in the control treatments. A particularly comprehensive impact was shown by the highest concentration of extracts from 'Pintor' $F$. ovina and 'Olivia' $F$. rubra. The influence of the concentrations of allelochemicals on their allelopathic potential is also confirmed by other authors. T a k a $\mathrm{h}$ a s hi et al. (1985), studying the allelopathic impact of water extracts of the sward residue of tropical grasses on the germination of L. multiflorum seeds, observed that the inhibition of seed germination of this species increased with an increase in the extract concentration. The study by Lipińska and $\mathrm{Harkot}$ (2005) also showed that the initial growth of the test species differed depending on the concentration of water extracts from $P$. pratensis leaves.

The effect of the allelopathic impact of extracts from plants is determined by the sensitivity of the test species (W a r d l e et al. 1992). Among the test species (acceptors), P. pratensis showed the greatest sensitivity at the seed germination stage. When subjected to extracts from all donors, seeds of this grass showed significantly poorer germination than when distilled water was applied. A considerable sensitivity was also demonstrated by $F$. ovina whose seed germination was significantly poorer under the influence of all extracts from 'Pintor' $F$. ovina, regardless of their concentration, and most donors at the concentration of $2.38 \%$ and $4.76 \%$. On the other hand, $F$. arundinacea seeds turned out to be the least sensitive because their germination was significantly poorer than in the control treatments only under the influence of the highest concentrations of the $F$. rubra cultivars studied.

During the root growth period, the greatest sensitivity was shown by A. capillaris; regardless of the donor, the growth process was inhibited by about 50\% relative to the control treatments. $P$. pratensis also proved to be very sensitive, particularly to extracts from 'Espro', 'Pintor' and 'Asterix' (root growth was inhibited by about 35\%). The acceptors mentioned above, particularly A. capillaris, also exhibited the highest sensitivity during the seedling growth period. The considerable sensitivity of $A$. capillaris and $P$. pratensis to the impact of water extracts may be linked to the small size of seeds of these species. Small seeds are more sensitive to the impact of allelochemicals than large ones (O le s z e k, 1992), and the disorders occurring at the initial stages of plant development may cause disruptions of many subsequent life processes (Sutherland et al. 1999). The lowest sensitivity to the impact of donors was exhibited by $F$. arundinacea. The response of the other acceptors to the allelopathic properties of aboveground vegetative shoots of grasses varied and depended on the tested parameter as well as on the origin of allelopathic substances and their current concentration. A similar response of acceptor species to specific allelopathic substances was confirmed, among other studies, by Bulut and De mir (2007). Seedlings of various species also showed various responses to the same extract concentrations in earlier studies by Li pińska and Harkot (2005). In their study, seeds of D. glomerata and Ph. pratense did not germinate at all in the treatments where the highest extract concentration was applied (4.76\%), while the germination of $F$. pratense seeds was significantly poorer than in the control treatments. In the study by Peters and $\mathrm{Zam}$ (1981), high concentrations of the water extract from $F$. arundinacea leaves inhibited the germination of $L$. corniculatus seeds significantly more than low concentrations, whereas the germination of $T$. pratense seeds was significantly inhibited both by high and low concentrations of these extracts.

\section{CONCLUSIONS}

1. The allelopathic potential of the tested donors varied and depended on the variety and concentration of water extracts as well as on the sensitivity of the acceptor.

2. 'Olivia' F. rubra and 'Pintor' F. ovina exhibited the highest allelopathic potential. A considerable 
potential was observed in the case of 'Asterix' $F$. arundinacea. The smallest adverse impact was exerted by 'Nimba' F. rubra.

3. The allelopathic potential of the individual cultivars varied within a particular species. Among the $F$. rubra cultivars, the greatest adverse impact was demonstrated by 'Olivia', then 'Areta', and the smallest by 'Nimba'. Among the F. ovina cultivars, 'Pintor' showed a stronger adverse impact than 'Espro'.

4. Regardless of the donor, the greatest adverse impact was exerted by the highest concentrations of extracts, resulting in poorer initial growth and development of the acceptors. Lower concentrations of all extracts primarily inhibited the growth of roots; all the parameters were inhibited only in the case of 'Pintor' $F$. ovina.

5. The sensitivity of the tested acceptors varied. Water extracts from aboveground vegetative shoots of the lawn grass cultivars caused the strongest response in A. capillaris and $P$. pratensis, resulting in poorer seed germination, shorter roots and smaller seedlings than in the control treatments. Considerable sensitivity was demonstrated by $F$. ovina and $L$. perenne, primarily during the root growth period. $F$. arundinacea showed the lowest sensitivity to the impact of the donors.

\section{Acknowledgements}

The study was conducted as part of the statutory activities of the Department of Grassland and Landscape Forming, University of Life Sciences in Lublin.

\section{Authors' contributions}

The following declarations about authors' contributions to the research have been made: HL, WH; laboratory research: MS; data analyses: HL, WH, MS; writing the manuscrpit: HL; comments on the manuscrpit HL, WH.

\section{REFERENCES}

Baghestani A., Lemieux C., Leroux G.D., Baziramakenga R., Simard R.R. 1999. Determination of allelochemicals in spring cereal cultivars of different competitiveness. Weed Sci. 47: 498-504.

Bertin C., Huang T., Schroeder F.C., Weston L.A., Jander G. 2005. Allelopathic Potential of Fine Leaf fescue: towards the elucidation of the mode of action of $m$-tyrosine in plant. American Society of Plant Biologists. Poster: Integrative plant physiology.

Blu m U. 1999. Designing laboratory plant debris-soil bioassays: some reflections. [In:] Principles and Practices in Plant Ecology: Allelochemical Interactions. Inderjit, K.M.M. Dakshini, Ch. L. Foy (ed.), CRC Press, 17-23.

Bulut Y., Demir M. 2007. The allelopathic effects of Scots Pine (Pinus sylvestris L.) leaf extracts on turf grass seed germination and seedling growth. Asian J. Chem. 19: 3169-3177.

Buta J.G., Spaulding D.W. 1987. Growth inhibiting compounds in tall fescue involved in allelopathy of turfgrasses - abscisic acid and phenolics. J. Chem. Ecol. 15 (5): 1629-1636.

Chung I.M., Miller D.A. 1995. Allelopathic influence of nine forage grass extracts on germination and growth of alfalfa. Agron. J. 87: 767-772. http://dx.doi. org/10.2134/agronj1995.00021962008700040026x

Czarnecki Z., Harkot W. 2002. Influence of cutting frequency on area sodding of lawn varieties of Lolium perenne. Grass. Sci. Pol. 5: 43-48 (in Polish, with English abstract).

Czyż H., Kitczak T. 2009. Dynamics of changes in lawn species composition in relation to type of substrate and type of mixture. Acta Agrophys. 167: 321-328 (in Polish, with English abstract).

Dorywalski J., Wojciechowicz M., Bartz J. 1964. Methods of seed evaluation. PWRiL, Warszawa (in. Polish).

Falkowski M. 1958. Meadows fatigue. Zesz. Probl. Post. Nauk Roln. 13: 7-31 (in. Polish).

Harkot W., Czarnecki Z., Lipińska H. 2000. Allelopathic influence of dead leaves of chosen species of lawn grass on initial development of Lolium perenne. [In:] W. Oleszek (ed.) Biochemical responses in environmental interactions. International Conference, IUNG Puławy 2-3 X. 2000, 57-58. (In Polish, with English abstract).

Harkot W., Czarnecki Z., Powroźnik M. 2006. Emergence and installment of selected lawn Grass varieties at different sowing dates. Zesz. Nauk. UP Wroc. 545: 111-120 (in. Polish, with English abstract).

Il a m A., Begum S. 2011. Evaluation of allelochemical effects of Hordeum vulgare extracts. Bangladesh Res. Publ. J. 5: 295-305.

Lickfeldt D.W., Voigt T.B., Branham B.E., Fermanian T.W. 2001. Evaluation in allelopathy in cool season turfgrass species. Inter. Turf. Soc. Res. J. 9: 1013-1018.

Lipińska H. 2005. Allelopathic effects of grasses and biodiversity of plant communities. Grass. Sci. Eur. 10: 380-383.

Lipińska H., Harkot W. 2005. Allelopathic effects of water leachates of Poa pratensis leaves on other grassland spp. Allelopathy J. 16: 251-260.

Lipińska H., Harkot W. 2007. Allelopathic activity of grassland species. Allelopathy J. 19: 3-36.

Majchrzak L. 2007. Cereals germination in the neighbourhood conditions of grain of Avena fatua L. and Festuca rubra L. - allelopathic aspect. Ann. UMCS, Sect. E, 62 (2): 185-192 (in. Polish, with English abstract). 
Oles ze k W. 1992.Techniques for study of allelopathy. Wiad. Bot. (Botanical News) 36: 17-25 (in. Polish).

Patrzałek A. 2000. Species and cultivars of grasses for special purposes and their utilisation. Grass. Sci. Pol. 3, 105-118 (in. Polish, with English abstract).

Peters E.J., Mohammed Zam A.H.B.1981.Allelopathic effects of tall fescue genotypes. Agron. J. 73: 56-58. http://dx.doi.org/10.2134/agronj1981.0002196200730 $0010013 \mathrm{x}$

Politycka B., Lipińska H. 2005. Pot cultures: simple tool and complex problem. Allelopathy J. 16: 47-62.

Smith A.E., Martin L.D. 1994. Allelopathic characteristics of three cool-season grass species in the forage ecosystem. Agron. J. 86: 243-246.

Sutherland, B.L., Hume, D.E. and Tapper, B.A. 1999. Allelopathic effects of endophyte-infected perennial ryegrass extracts on white clover seedlings. New Zealand J. Agric. Res. 42: 19-26. http://dx.doi.org/10.1 080/00288233.1999.9513349

Takahashi Y., Otani I., Uozumi S., Yoden Y., Igarashi R. 1988. Studies on the allelopathie interactions among some grassland species. I. Effect of root exudates from some grass and legume species on the growth of their own species and other species. J. Japan. Soc. Grass. Sci. 33 (4): 338-344.

Wardle D.A., Nicholson K.S., Rahman A. 1992. Comparison of osmotic and allelopathic effects of grass leaf extracts on grass seed germination and radicle elongation. Plant and Soil 140 (2): 315-319. http://dx.doi. org/10.1007/BF00010609

Wolski K. Szymura M., Szymura T., Gierula A., Sokulska D. 2006. Cereals germination in the neighbourhood conditions of grain of Avena fatua L. and Festuca rubra L. - allelopathic aspect. Zesz. Naukowe UP we Wrocławiu. Rolnictwo LXXXVIII, 545, 301-313 (in Polish, with English abstract).

\section{Wpływ wodnych wyciągów z liści \\ Festuca rubra, F. ovina i $F$. arundinacea na początkowy wzrost i rozwój innych gatunków traw}

\section{Streszczenie}

Jednym z najmniej poznanych czynników decydujących o stabilności muraw trawnikowych są allelopatyczne oddziaływania roślin. Bogatym źródłem substancji allelopatycznych są liście. Substancje te, wymywane przez deszcz czy krople rosy bądź uwalniane w czasie rozkładu biomasy, mogą oddziaływać na rośliny. W praktyce często ścięta murawa jest pozostawiana na powierzchni trawnika i może allelopatycznie oddziaływać na odrastające rośliny. Efekt oddziaływania uwolnionych allelozwiązków zależy od wielu czynników, w tym od ich stężenia. Zatem, aby utrzymać wysokie walory użytkowe trawnika, niezbędne są informacje o krytycznych koncentracjach allelozwiązków hamujących wzrost i rozwój roślin. Stąd podjęto badania laboratoryjne (na płytkach Petriego), w których oceniano wpływ różnych stężeń wodnych wyciągów z liści wybranych gazonowych odmian traw. Donorami były odmiany: 'Areta', 'Nimba', 'Olivia' (Festuca rubra); 'Espro', 'Pintor' ( $F$. ovina) oraz 'Asterix' $(F$. arundinacea), natomiast akceptorami: 'Niwa' (Agrostis capillaris), 'Asterix' ( $F$. arundinacea), 'Espro' (F. ovina), 'Areta' (F. rubra), 'Stadion' (Lolium perenne) i 'Bila' (Poa pratensis) - gatunki często wysiewane na trawniki. Kontrolę stanowiły obiekty, w których podłoże zwilżano wodą destylowaną.

Badania wykazały, że oddziaływania wodnych wyciągów z liści były zróżnicowane w zależności od ich stężenia i donora (odmiany) oraz wrażliwości akceptora (rośliny testowej). W porównaniu do obiektów kontrolnych największy ujemny wpływ powodowały wodne wyciągi z liści odmian Olivia (F.rubra) i 'Pintor' ( $F$. ovina), a następnie 'Asterix' ( $F$. arundinacea). Wśród akceptorów, największą wrażliwością na obecność allelozwiązków charakteryzowała się A. capillaris, zaś najmniejszą $F$. arundinacea. 